\title{
Frictional Granular Mechanics: A Variational Approach
}

\author{
R. Holtzman ${ }^{1}$, D. B. Silin ${ }^{2}$, T. W. Patzek ${ }^{1,3}$ \\ ${ }^{1}$ Department of Civil and Environmental Engineering, University of California, Berkeley, CA 94720, USA \\ ${ }^{2}$ Lawrence Berkeley National Laboratory, 1 Cyclotron Road, MS 90R1116, Berkeley, CA 94720, USA \\ ${ }^{3}$ Department of Petroleum \& Geosystems Engineering, University of Texas, Austin, TX 78712, USA
}

\section{SUMMARY}

The mechanical properties of a cohesionless granular material are evaluated from grain-scale simulations. Intergranular interactions, including friction and sliding, are modeled by a set of contact rules based on the theories of Hertz, Mindlin, and Deresiewicz. A computer generated, three-dimensional, irregular pack of spherical grains is loaded by incremental displacement of its boundaries. Deformation is described by a sequence of static equilibrium configurations of the pack. A variational approach is employed to find the equilibrium configurations by minimizing the total work against the intergranular loads. Effective elastic moduli are evaluated from the intergranular forces and the deformation of the pack. Good agreement between the computed and measured moduli, achieved with no adjustment of material parameters, establishes the physical soundness of the proposed model.

key words: micromechanics; grain-scale simulations; intergranular friction; quasi-static model; effective elastic moduli; principle of least work

\section{INTRODUCTION}

In this paper, we present a micromechanical model of a cohesionless particulate material, evaluating its mechanical properties by numerical simulations. The abundance of related applications and their large socioeconomic impact have motivated intensive research in the field of granular mechanics (see the references within [1, 2]). Technical difficulties associated with grain-scale measurements, together with the shortcomings of continuum macroscopic models, make micromechanical simulations a prominent tool.

Obtaining the equilibrium configurations of a grain assembly requires solving a large nonlinear system of equations. In most micromechanical models, e.g. the discrete element method (DEM) [3], these equations are solved by explicit numerical integration over time. Constraints imposed on the time step make DEM simulations time-consuming $[4,5]$. To enhance convergence to a static equilibrium, a damping parameter is often introduced, and its

Correspondence to: Department of Civil and Environmental Engineering, Massachusetts Institute of Technology, 77 Massachusetts Avenue, Building 48, Cambridge, MA 02139, USA Contract/grant sponsor: NETL under DOE; Contract/Grant Number: \#DE-FC26-05NT42664, \#DE-AC0205CH11231 
value together with other grain-scale parameters are adjusted [6-10], sometimes to unrealistic values. Such adjustments affect the computed mechanical properties of the pack, and may result in "over-damping" of the grain motion, leaving a substantial number of grains out of a stable equilibrium [11]. These difficulties have motivated development of implicit methods. For example, a model of a pack of disks based on the discontinuous deformation analysis (DDA) method [12] was proposed in [11]. DDA employs minimization of potential energy and the penalty method to solve for the displacements of blocks. Consequently, a system of linear equations is solved at each time step [11].

A computational technique to simulate deformation of a granular material and evaluate its mechanical properties, denoted hereafter as Quasi-Static Granular Model (QuSGM), has been presented in [1]. In QuSGM, a three-dimensional (3D) irregular pack of spherical grains is loaded by incremental displacement of its boundaries. Deformation is described by a sequence of equilibrium configurations, and time integration is avoided. According to the principle of minimum potential energy [13], each equilibrium configuration is sought by minimizing the potential energy of the pack with respect to the grain displacements using a modified conjugate gradient method. The macroscopic stress, strain, and elastic moduli are evaluated from the intergranular forces and the deformation of the pack.

The model in [1], denoted as "frictionless," neglects intergranular friction, thus accounting for normal contact forces only. These forces are evaluated by the Hertzian contact model, which, for small deformations of relatively stiff particles, has been verified by experiments and numerical simulations $[14,15]$. The results in [1] confirm that the resistance of a grain pack to volumetric compaction, characterized by the bulk modulus, mainly depends on the normal contact forces $[6,16]$, and that the assumption of grain-scale elasticity can capture inelastic deformation of a granular pack [17]. For instance, micromechanical analysis reveals the mechanisms responsible for hysteresis, strain hardening, and stress-induced anisotropy [1].

While the model in [1] supports the idea that microscopic friction is not the sole mechanism of macroscopic shear resistance [10], it underestimates the shear modulus. We associate this deficiency, in part, with the assumption of negligible intergranular friction [1]. In this paper, QuSGM is extended to account for friction.

Friction resists relative displacements and rotations of grains through shear forces and torsional couples ("frictional loads") at the contacts. For small grain deformations, the models of Mindlin and Deresiewicz [18, 19] adequately describe intergranular shear ([14, 20, 21] and the references therein) and torsion [14,22]. Slip between the grain surfaces dissipates energy, making load-displacement relations path-dependent. In $[18,19]$, this path-dependency is modeled by tracking the load history in incremental steps [14,23]. At each step, the contact compliance evaluation accounts for the load increment, the reference load prior to the application of that increment, and the maximum load experienced prior to unloading or reloading of that contact. The complexity of the Mindlin and Deresiewicz models motivated the development of simpler approaches to computation of the grain pack response [20,23]. For instance, in $[20,24]$ the tangential force is computed by accounting for a reduced number of load scenarios. Since the contribution of torsional couples to the averaged stress in cohesionless materials is negligible $[2,25]$, intergranular torsion is often neglected in simpler models.

In this study, the Mindlin and Deresiewicz models are simplified through a set of incrementally path-independent constitutive relations. The capability to predict the mechanical properties of a granular sample from its grain properties, with no adjustment of parameters, demonstrates the physical soundness of our approach. 
The outline of this paper is as follows. Section 2 describes the model and simulation procedure. Section 3 presents the results of simulations. The values of the model parameters used in the simulations are specified in Section 3.1. The generation of a numerical grain pack is discussed in Section 3.2. In Section 3.3, the model is verified against experimental data. The sensitivity of the model's predictions to the initial packing, the size of the incremental load step, and the intergranular friction coefficient are examined in Sections 3.4-3.6. Section 4 provides summary and conclusions. The numerical algorithm is outlined in Appendix A.

\section{A MICROMECHANICAL MODEL OF A GRANULAR MATERIAL}

A granular sample is modeled as a 3D irregular packing of spherical grains, bounded by the solid walls of a rectangular container. Simulations begin with a sufficiently-dense irregular arrangement, denoted hereafter as the initial configuration. An irregular pack is used since a regular arrangement, uncommon in natural materials [26,27], deforms in a fundamentally different manner than an irregular one $[28,29]$.

We use a quasi-static model, describing deformation as a sequence of static equilibrium configurations. Starting from a reference configuration, a load is applied by incremental displacement of the pack's boundaries. Following such perturbation, the grains deform and rearrange into a new ("current") equilibrium configuration. The current configuration of the previous step serves as the reference configuration of the next step. In the following sections we discuss the characterization of a grain pack, the constitutive relations that are used to compute the intergranular loads, a methodology of obtaining the equilibrium configurations, and the evaluation of the macroscopic parameters.

\subsection{Characterization of a grain pack}

This section provides a quantitative description of the configuration of a grain pack. Each equilibrium configuration is characterized by the translations and rotations of the grains relative to a reference configuration, that satisfy the force and moment balance equations for each grain. While the pack geometry is fully described by the radii and centers of the grains, the grain rotations are required to compute the intergranular shear and torsional loads. Given a reference configuration, a pack of $N$ grains has $6 N$ degrees of freedom: the displacements of the grain centers and the grain rotations.

Labeling each grain with a single index, $i=1,2, \ldots, \mathrm{N}$, we denote the displacement of grain $i$ by $\boldsymbol{u}_{i}=\boldsymbol{r}_{i}-\boldsymbol{r}_{i}^{0}$, where $\boldsymbol{r}_{i}$ is the radius-vector of the grain center, and superscript 0 denotes the reference configuration, $c f$. Figure 1 (a). The vector $\boldsymbol{\Omega}_{i}$ denotes the rotation of grain $i$ by an angle $\left\|\boldsymbol{\Omega}_{i}\right\|$, around an axis passing through the grain's center and directed along $\boldsymbol{\Omega}_{i}$, using the right-hand convention. Here, $\|\boldsymbol{\xi}\|=(\boldsymbol{\xi} \cdot \boldsymbol{\xi})^{1 / 2}$ is the magnitude of a vector $\boldsymbol{\xi}$. To make the units of the variables uniform, the rotation of each grain is multiplied by its radius, $R_{i}$. Thus, a vector of generalized coordinates, $\boldsymbol{\theta}=\left[\begin{array}{llllll}\boldsymbol{u}_{1} & \ldots & \boldsymbol{u}_{N} & \boldsymbol{\Omega}_{1} R_{1} & \ldots & \boldsymbol{\Omega}_{N} R_{N}\end{array}\right]$, where $\boldsymbol{u}_{i}$ and $\boldsymbol{\Omega}_{i}$ are row vectors, characterizes the grain pack configuration.

This study considers a dense pack of relatively stiff grains, analogous to quartz. In such a pack, grains exhibit very little damage unless exceedingly high stresses are applied $[26,27]$. Thus, the grain deformations are often assumed to be small and localized near the contacts [14]. In our model, each grain consists of homogeneous and isotropic elastic material with Young's 
modulus and Poisson's ratio denoted by $E_{i}$ and $\nu_{i}$, respectively. The density of the grain material, $\rho_{i}$, has little effect on the equilibrium configuration; while gravity stabilizes a loose packing, its effect is negligible relative to the contact forces in a dense, stressed pack.

The pack's boundaries are represented by elastic planar walls which interact with the outermost grains. We align the domain with a Cartesian coordinate system, labeling each boundary with a single index, $w=1,2, \ldots, 6$. The position of a planar boundary $w$ is determined by its orientation, which is specified by an inward unit normal, $\hat{\boldsymbol{n}}_{w}$, and an arbitrary point on the plane, $\boldsymbol{x}_{w}$, see Figure 1(a). The displacement of the boundary is denoted by $\boldsymbol{u}_{w}$. The Young's modulus and Poisson's ratio of the boundary material are denoted by $E_{w}$ and $\nu_{w}$.

(a)

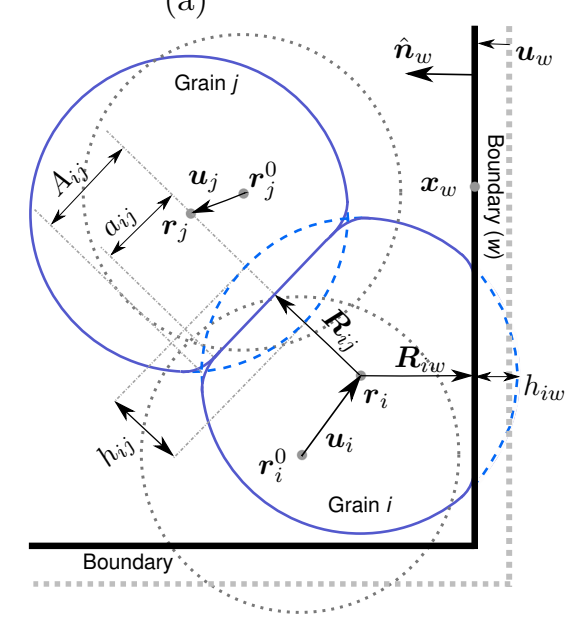

(b)

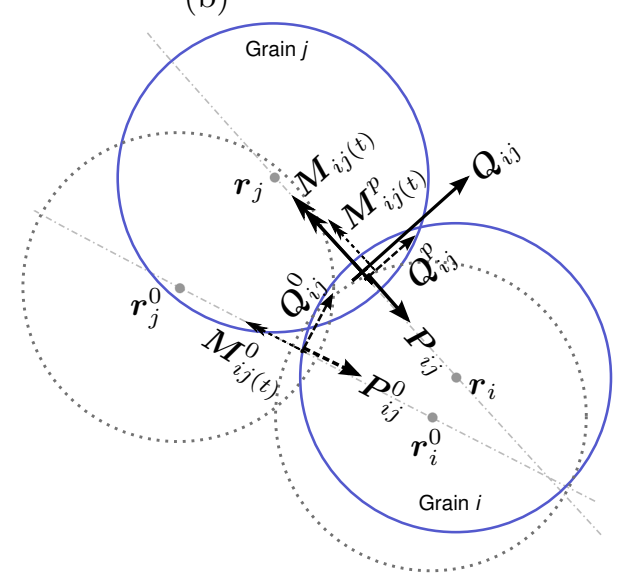

Figure 1. (a) Schematic description of the contact geometry. The reference configuration of the grains and boundaries is marked with grey dotted lines. The dashed lines show the shapes of the undeformed grains in the current configuration. (b) The loads on grain $i$ due to its contact with grain $j$, at the reference and current configuration, marked by dashed and solid arrows, respectively. The reference loads are projected onto the current contact area to account for its rotation.

\subsection{Intergranular constitutive relations}

2.2.1. Normal contact forces. At each contact, we resolve the contact force into normal and tangential components. Following $[18,19]$, we assume that the normal component is independent of the other load components, and evaluate it using the Hertzian model. This assumption has been justified analytically for the case of identical grains [14], and numerically for dissimilar grain properties [15]. For a pair of spherical grains, $i$ and $j$, the contact area is modeled as a planar disk of radius $a_{i j}$,

$$
a_{i j}=\left(R_{i j}^{*} h_{i j}\right)^{1 / 2}
$$

The magnitude of the normal compressive force acting at the contact is

$$
\left\|\boldsymbol{P}_{i j}\right\|=\frac{4}{3} E_{i j}^{*}\left(R_{i j}^{*}\right)^{1 / 2}\left(h_{i j}\right)^{3 / 2}
$$


where $R_{i j}^{*}=\left(1 / R_{i}+1 / R_{j}\right)^{-1}$ and $E^{*}=\left[\left(1-\nu_{i}^{2}\right) / E_{i}+\left(1-\nu_{j}^{2}\right) / E_{j}\right]^{-1}$ are the effective radius and elastic coefficient associated with this contact [14]. The above equations are also applicable to a grain-boundary contact by assigning an infinite radius to the planar wall, $R_{i w}^{*}=R_{i}$. The normal compression is quantified by the mutual approach, $h_{i j} \geq 0$,

$$
\begin{aligned}
h_{i j} & =R_{i}+R_{j}-\left\|\boldsymbol{r}_{i j}\right\| & & \text { for a grain-grain contact } \\
h_{i w} & =R_{i}-\left(\boldsymbol{r}_{i}-\boldsymbol{x}_{w}\right) \cdot \hat{\boldsymbol{n}}_{w} & & \text { for a grain-boundary contact }
\end{aligned}
$$

where $\boldsymbol{r}_{i j}=\boldsymbol{r}_{i}-\boldsymbol{r}_{j}$, see Figure 1(a). The normal forces acting on grain $i$ at the contact with another grain $j$ and a wall $w$ are directed along $\boldsymbol{r}_{i j}$ and $\hat{\boldsymbol{n}}_{w}$, respectively. Thus, a zero moment relative to the center of the grain is associated with the normal force.

2.2.2. Tangential contact forces. The shear ("tangential") force associated with frictional resistance is computed from the relative lateral displacements and rotations of a pair of contacting grains, through a constitutive relation based on the Mindlin-Deresiewicz model [18].

Consider a reference configuration with a pair of contacting grains, $i$ and $j$. Grain $i$ is loaded by a force $\boldsymbol{Q}_{i j}^{0}$ which is tangential to the contact plane, and a normal force, $\boldsymbol{P}_{i j}^{0}$, $c f$. Figure 1 (b). We assume that incremental boundary displacements cause small linear and angular grain displacements. These displacements correspond to a relative tangential displacement of grain $i$ with respect to $j, \boldsymbol{u}_{i j(s)}$, which alters the tangential force. The tangential force, $\boldsymbol{Q}_{i j}$, is calculated by incrementing the unperturbed value,

$$
\boldsymbol{Q}_{i j}=\boldsymbol{Q}_{i j}^{p}-k_{i j(s)} \boldsymbol{u}_{i j(s)}
$$

where $k_{i j(s)}$ denotes the shear resistance ("stiffness") of the contact. Subscript $(s)$ denotes a parameter associated with shear. Following [30], the reorientation of the contact interface is accounted for by aligning the force $\boldsymbol{Q}_{i j}^{0}$ with the direction of the current contact area, $\boldsymbol{Q}_{i j}^{p}=\left\|\boldsymbol{Q}_{i j}^{0}\right\| \hat{\boldsymbol{t}}_{i j}$, see Figure 1(b). The unit vector $\hat{\boldsymbol{t}}_{i j}=\boldsymbol{T}_{i j} \boldsymbol{Q}_{i j}^{0} /\left\|\boldsymbol{T}_{i j} \boldsymbol{Q}_{i j}^{0}\right\|$ denotes the direction of the rotated force, where $\boldsymbol{T}_{i j}=\hat{\boldsymbol{I}}-\hat{\boldsymbol{r}}_{i j} \otimes \hat{\boldsymbol{r}}_{i j}$ and $\hat{\boldsymbol{r}}_{i j}=\boldsymbol{r}_{i j} /\left\|\boldsymbol{r}_{i j}\right\|$. Here, $\hat{\boldsymbol{I}}$ is a second order identity tensor and $\otimes$ denotes the tensor product. For a contact with a boundary $w$, $\boldsymbol{T}_{i w}=\hat{\boldsymbol{I}}-\hat{\boldsymbol{n}}_{w} \otimes \hat{\boldsymbol{n}}_{w}$. The relative tangential displacement is

$$
\begin{aligned}
\boldsymbol{u}_{i j(s)} & =\boldsymbol{T}_{i j}\left(\boldsymbol{u}_{i}-\boldsymbol{u}_{j}\right)+\boldsymbol{\Omega}_{i} \times \boldsymbol{R}_{i j}-\boldsymbol{\Omega}_{j} \times \boldsymbol{R}_{j i} & & \text { for a grain-grain contact } \\
\boldsymbol{u}_{i w(s)} & =\boldsymbol{T}_{i w}\left(\boldsymbol{u}_{i}-\boldsymbol{u}_{w}\right)+\boldsymbol{\Omega}_{i} \times \boldsymbol{R}_{i w} & & \text { for a grain-boundary contact }
\end{aligned}
$$

The radius-vectors $\boldsymbol{R}_{i j}=-\hat{\boldsymbol{r}}_{i j}\left(R_{i}^{2}-A_{i j}^{2}\right)^{1 / 2}$ and $\boldsymbol{R}_{i w}=-\hat{\boldsymbol{n}}_{w}\left(R_{i}-h_{i w}\right)$ connect the center of grain $i$ to the center of the contact area with another grain $j$ and a boundary $w$, respectively. The intersection of the undeformed grain surfaces defines a disk of radius $A_{i j}=\left[\left(R_{i}-R_{j}-r_{i j}\right)\left(-R_{i}+R_{j}-r_{i j}\right)\left(R_{i}+R_{j}-r_{i j}\right)\left(R_{i}+R_{j}+r_{i j}\right)\right]^{1 / 2} /\left(2 r_{i j}\right)$, where $r_{i j}=\left\|\boldsymbol{r}_{i j}\right\|$; see Figure 1(a). The vector product is denoted by $\times$.

If intergranular slip occurs, the force-displacement relation becomes path-dependent [18]. In the Mindlin-Deresiewicz model [18], slip is accounted for by varying the shear stiffness with the current load and the load history. Here, we formulate a linear, path-independent, incremental force-displacement relation. The linearization is justified by the assumption of small grain displacements [31]. With this linear approximation, we neglect the effect of the normal force increment on $\boldsymbol{Q}_{i j}$, so that $\boldsymbol{Q}_{i j}$ is a function of the reference value, $\boldsymbol{P}_{i j}^{0}$. At each load increment, we assume that a contact experiences either perfect stick ("no-slip") or 
complete slip ("sliding"). According to the Coulomb friction model, the tangential force cannot exceed $Q_{i j}^{\max }=\mu_{i j}\left\|\boldsymbol{P}_{i j}\right\|$, where $\mu_{i j}$ is the intergranular friction coefficient. Sliding occurs when the shear force reaches this threshold, and the force magnitude becomes independent of the stiffness, $\left\|\boldsymbol{Q}_{i j}\right\|=Q_{i j}^{\max }$. To model this hysteretic behavior, we evaluate the stiffness by

$$
k_{i j(s)}= \begin{cases}k_{i j(s)}^{*} & \left\|\boldsymbol{Q}_{i j}^{*}\right\| \leq Q_{i j}^{\max } \\ k_{i j(s)}^{\prime} & \left\|\boldsymbol{Q}_{i j}^{*}\right\|>Q_{i j}^{\max }\end{cases}
$$

where $\boldsymbol{Q}_{i j}^{*}=\boldsymbol{Q}_{i j}^{p}-k_{i j(s)}^{*} \boldsymbol{u}_{i j(s)}, c f$. Eq. (4), and

$$
\begin{aligned}
& k_{i j(s)}^{*}=8 a_{i j}\left(\frac{2-\nu_{i}}{G_{i}}+\frac{2-\nu_{j}}{G_{j}}\right)^{-1} \\
& k_{i j(s)}^{\prime}=\frac{1}{\left\|\boldsymbol{u}_{i j(s)}\right\|^{2}}\left\{\boldsymbol{Q}_{i j}^{p} \cdot \boldsymbol{u}_{i j(s)}+\left[\left(\boldsymbol{Q}_{i j}^{p} \cdot \boldsymbol{u}_{i j(s)}\right)^{2}+\left(\left(Q_{i j}^{\text {max }}\right)^{2}-\left\|\boldsymbol{Q}_{i j}^{p}\right\|^{2}\right)\left\|\boldsymbol{u}_{i j(s)}\right\|^{2}\right]^{1 / 2}\right\}
\end{aligned}
$$

Here, $G_{i}$ is the shear modulus of the material of grain $i$. The value of $k_{i j(s)}^{*}$ is predicted in [18] for the case of negligible slip. Negligible slip is expected at the onset of either loading or unloading, as the tangential force either increases from zero or decreases after a monotonic loading, $c f$. Figure 2. Sliding is accounted for by reducing the stiffness as the magnitude of the tangential force approaches $Q_{i j}^{\max }$. The reduced value, $k_{i j(s)}^{\prime}$, makes the shear force equal to $Q_{i j}^{\max }$; thus, $k_{i j(s)}^{\prime}$ vanishes if the sliding threshold has been reached. Physically, the stiffness coefficient is non-negative. Thus, we put $k_{i j(s)}^{\prime}=0$ if the term inside the square brackets in Eq. (7b) becomes negative.

With the linear approximation, we use the unperturbed force, $\boldsymbol{P}_{i j}^{0}$, rather than $\boldsymbol{P}_{i j}$ in Eqs. (6)-(7). Similarly, we use the corresponding unperturbed values of the radius of the disk of intersection, $A_{i j}^{0}$, and the contact radius, $a_{i j}^{0}$. To justify the latter we expand the expression in Eq. (1) as a Taylor series near $h_{i j}=h_{i j}^{0}$, noting that the linear term in the expansion can be neglected since $\left|h_{i j}-h_{i j}^{0}\right| / h_{i j}^{0} \ll 1$. Here, $h_{i j}^{0}$ is the reference value of $h_{i j}$.

Finally, the force $\boldsymbol{Q}_{i j}$ is associated with a moment relative to the center of grain $i$,

$$
\boldsymbol{M}_{i j(s)}=\boldsymbol{R}_{i j} \times \boldsymbol{Q}_{i j}
$$

2.2.3. Torsional couples. Relative torsion between a pair of grains creates a torsional couple at their contact $[19,32]$. In Section 2.4, we demonstrate that the contribution of the intergranular torsion to the macroscopic stress is negligible. However, in a cemented grain pack, torsion may determine the strength of each contact [33]. To enable future extension of the model to account for cemented grains, a torque-rotation relation based on the model in [19] is formulated below, using an approach similar to that in Section 2.2.2.

Consider a pair of grains, $i$ and $j$, loaded by a torsional couple, $\boldsymbol{M}_{i j(t)}^{0}$, and a normal force, $\boldsymbol{P}_{i j}^{0}$. Subscript $(t)$ denotes a parameter associated with torsion. Following a perturbation of the boundaries, relative torsion between the grains, $\boldsymbol{\Omega}_{i j(t)}$, modifies the torque,

$$
\boldsymbol{M}_{i j(t)}=\boldsymbol{M}_{i j(t)}^{p}-k_{i j(t)} \boldsymbol{\Omega}_{i j(t)}
$$

where the stiffness $k_{i j(t)}$ describes the resistance of the contact to torsion. The effect of the rotation of the contact interface is modeled by $\boldsymbol{M}_{i j(t)}^{p}=\left\|\boldsymbol{M}_{i j(t)}^{0}\right\| \operatorname{sgn}\left(\boldsymbol{M}_{i j(t)}^{0} \cdot \hat{\boldsymbol{r}}_{i j}\right) \hat{\boldsymbol{r}}_{i j}$, see 

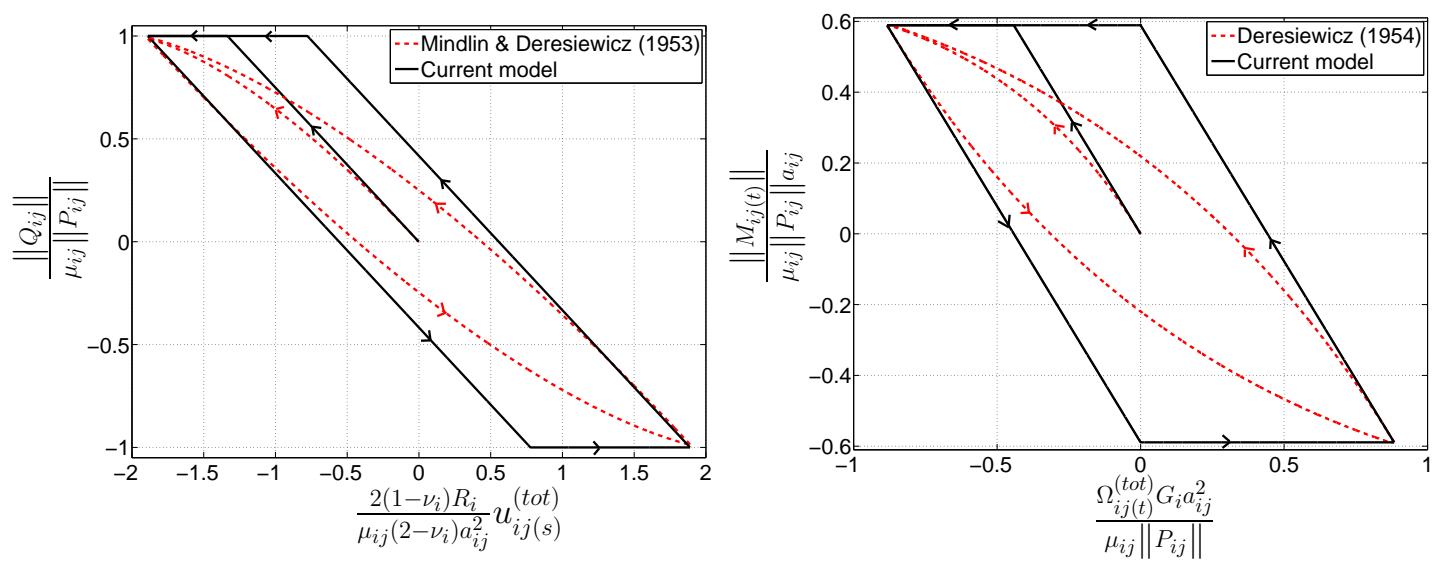

Figure 2. Left: the intergranular shear force, $\left\|Q_{i j}\right\|$, vs. the relative shear displacement since contact is established, $u_{i j(s)}^{(t o t)}$. Right: the intergranular torsional couple, $\left\|M_{i j(t)}\right\|$, vs. the relative torsion since contact is established, $\Omega_{i j(t)}^{(t o t)}$. The loads are computed using the models in Sections 2.2.2-2.2.3 and in [18] and [19], for a loading-unloading-reloading cycle (see arrows) while fixing the normal force. The plot shows the magnitudes of the respective vectorial quantities, normalized to a non-dimensional form. The shear and torsional stiffness, $k_{i j(s)}$ and $k_{i j(t)}$, correspond to the slope of the lines.

Figure 1(b). For a contact with a boundary $w, \boldsymbol{M}_{i w(t)}^{p}=\left\|\boldsymbol{M}_{i w(t)}^{0}\right\| \operatorname{sgn}\left(\boldsymbol{M}_{i w(t)}^{0} \cdot \hat{\boldsymbol{n}}_{w}\right) \hat{\boldsymbol{n}}_{w}$. Here, sgn denotes the sign function. The relative torsion is evaluated by

$$
\begin{aligned}
\boldsymbol{\Omega}_{i j(t)} & =\left[\left(\boldsymbol{\Omega}_{i}-\boldsymbol{\Omega}_{j}\right) \cdot \hat{\boldsymbol{r}}_{i j}\right] \hat{\boldsymbol{r}}_{i j} & & \text { for a grain-grain contact } \\
\boldsymbol{\Omega}_{i w(t)} & =\left(\boldsymbol{\Omega}_{i} \cdot \hat{\boldsymbol{n}}_{w}\right) \hat{\boldsymbol{n}}_{w} & & \text { for a grain-boundary contact }
\end{aligned}
$$

Slip may occur in a circumferential direction, making the torque-rotation relation pathdependent [19]. Linearization of the torque-rotation relation decouples the torque from the normal force increment. At each load increment, a path-independent torque-rotation relation is formulated by assuming either no-slip or sliding. The torque magnitude is limited by $M_{i j(t)}^{\max }=(3 \pi / 16) a_{i j} \mu_{i j}\left\|\boldsymbol{P}_{i j}\right\|$, which is the expected value in sliding [32], by defining

$$
k_{i j(t)}= \begin{cases}k_{i j(t)}^{*} & \left\|\boldsymbol{M}_{i j(t)}^{*}\right\| \leq M_{i j(t)}^{\max } \\ k_{i j(t)}^{\prime} & \left\|\boldsymbol{M}_{i j(t)}^{*}\right\|>M_{i j(t)}^{\max }\end{cases}
$$

where $\boldsymbol{M}_{i j(t)}^{*}=\boldsymbol{M}_{i j(t)}^{p}-k_{i j(t)}^{*} \boldsymbol{\Omega}_{i j(t)}, c f$. Eq. (9), and

$$
\begin{aligned}
k_{i j(t)}^{*} & =\frac{16}{3} a_{i j}{ }^{3}\left(\frac{1}{G_{i}}+\frac{1}{G_{j}}\right)^{-1} \\
k_{i j(t)}^{\prime} & =\frac{1}{\left\|\boldsymbol{\Omega}_{i j(t)}\right\|^{2}}\left\{\boldsymbol{M}_{i j(t)}^{p} \cdot \boldsymbol{\Omega}_{i j(t)}+\left[\left(\boldsymbol{M}_{i j(t)}^{p} \cdot \boldsymbol{\Omega}_{i j(t)}\right)^{2}+\right.\right. \\
& \left.\left.\left(\left(M_{i j(t)}^{\max }\right)^{2}-\left\|\boldsymbol{M}_{i j(t)}^{p}\right\|^{2}\right)\left\|\boldsymbol{\Omega}_{i j(t)}\right\|^{2}\right]^{1 / 2}\right\}
\end{aligned}
$$


Here, $k_{i j(t)}^{*}$ is the stiffness in the case of negligible slip, expected at the onset of torsional loading or unloading [19], see Figure 2. To enforce the torsional threshold, the stiffness is reduced to $k_{i j(t)}^{\prime}$, which vanishes at sliding. Similar to Eq. $(7 \mathrm{~b})$, we put $k_{i j(t)}^{\prime}=0$ if the term inside the square brackets in Eq. (12b) becomes negative. With the linear approximation, the unperturbed values $\boldsymbol{P}_{i j}^{0}$ and $a_{i j}^{0}$ are used in Eqs. (11)-(12) to determine $M_{i j(t)}^{\max }$ and $k_{i j(t)}$.

\subsection{Equilibrium configurations}

This section describes a methodology to obtain an equilibrium configuration. Each configuration is characterized by a set of generalized coordinates, $\boldsymbol{\theta}$, which satisfy the force and moment balance for each grain,

$$
\begin{gathered}
\boldsymbol{F}_{i}=\sum_{j=1}^{N_{g}^{i}}\left(\boldsymbol{P}_{i j}+\boldsymbol{Q}_{i j}\right)+\sum_{w=1}^{N_{b}^{i}}\left(\boldsymbol{P}_{i w}+\boldsymbol{Q}_{i w}\right)-m_{i} g \hat{\boldsymbol{e}}_{z}=0 \\
\boldsymbol{M}_{i}=\sum_{j=1}^{N_{g}^{i}}\left(\boldsymbol{M}_{i j(s)}+\boldsymbol{M}_{i j(t)}\right)+\sum_{w=1}^{N_{b}^{i}}\left(\boldsymbol{M}_{i w(s)}+\boldsymbol{M}_{i w(t)}\right)=0
\end{gathered}
$$

The sums of forces and moments acting on grain $i$ are denoted by $\boldsymbol{F}_{i}$ and $\boldsymbol{M}_{i}$, respectively. Employing a variational approach, each equilibrium configuration is obtained by finding a local minimum of an energy functional, $\Pi$, with respect to $\boldsymbol{\theta}$. In the absence of friction, $\Pi$ equals the total potential energy of the pack [1]. Here, the total energy of the pack is not potential. By the principle of least work [13], we seek a minimum of the mechanical work done against the intergranular loads following a perturbation of the boundaries,

$$
\Pi=-\sum_{i=1}^{N}\left\{\frac{1}{2} \sum_{j=1}^{N_{g}^{i}} W_{i j}+\sum_{w=1}^{N_{b}^{i}} W_{i w}-m_{i} g\left(\boldsymbol{u}_{i} \cdot \hat{\boldsymbol{e}}_{z}\right)\right\}
$$

where $g$ is the acceleration due to gravity, $\hat{\boldsymbol{e}}_{z}$ is a unit vector pointing opposite to the direction of gravity, and the grain mass is $m_{i}=(4 / 3) \pi R_{i}^{3} \rho_{i}$. We denote by $N_{g}^{i}$ and $N_{b}^{i}$ the number of contacts of grain $i$ with other grains and with boundaries, respectively, so that $N^{i}=N_{g}^{i}+N_{b}^{i}$ is the coordination number. In Eq. (14), $W_{i j}$ is the work done against the loads acting on grain $i$ due to its contact with grain $j$,

$$
W_{i j}=W_{i j(n)}+W_{i j(s)}+W_{i j(t)}
$$

Here $W_{i j(n)}, W_{i j(s)}$, and $W_{i j(t)}$ are the mechanical works against the normal and tangential contact forces, and torsional couples, respectively. We evaluate $W_{i j(n)}$ from the increment of elastic strain energy associated with the normal compression [34],

$$
W_{i j(n)}=-(8 / 15) E_{i j}^{*}\left(R_{i j}^{*}\right)^{1 / 2}\left[\left(h_{i j}\right)^{5 / 2}-\left(h_{i j}^{0}\right)^{5 / 2}\right]
$$

The works against the shear and torsional loads are evaluated by integrating these loads over the relative shear and torsional displacements, respectively. These integrals are evaluated numerically using a midpoint rectangular rule,

$$
\begin{aligned}
& W_{i j(s)}=\boldsymbol{Q}_{i j}^{p} \cdot \boldsymbol{u}_{i j(s)}-\frac{1}{2} k_{i j(s)}\left\|\boldsymbol{u}_{i j(s)}\right\|^{2} \\
& W_{i j(t)}=\boldsymbol{M}_{i j(t)}^{p} \cdot \boldsymbol{\Omega}_{i j(t)}-\frac{1}{2} k_{i j(t)}\left\|\boldsymbol{\Omega}_{i j(t)}\right\|^{2}
\end{aligned}
$$


In sliding, the stiffness vanishes and Eqs. (17) evaluate the work of fixed inelastic force and torque with respect to the displacements. Following [20], we assume that friction is the only means of energy dissipation.

We obtain a local minimum of $\Pi$ numerically (see Appendix A). At a minimum, the gradient of $\Pi$ with respect to the generalized coordinates, $\boldsymbol{\theta}$,

$$
\nabla_{\boldsymbol{\theta}} \Pi=-\left[\begin{array}{llllll}
\boldsymbol{F}_{1} & \ldots & \boldsymbol{F}_{N} & \boldsymbol{M}_{1} / R_{1} & \ldots & \boldsymbol{M}_{N} / R_{N}
\end{array}\right]
$$

vanishes. Thus, the zero gradient of $\Pi$ means the balance of forces and moments.

\subsection{Macroscopic parameters}

For each equilibrium configuration, we evaluate the average strain $(\boldsymbol{\epsilon})$ and stress $(\boldsymbol{\sigma})$ within the sample, and the effective elastic moduli. The evaluation procedure is summarized below.

2.4.1. Strain. The strain is evaluated from the boundary displacements [1]. The normal strain in the $l$-direction $(l=1,2,3)$ is $\left(L_{l}-L_{l}^{\sigma=0}\right) / L_{l}^{\sigma=0}$, where $L_{l}$ and $L_{l}^{\sigma=0}$ are the length of the domain in that direction, in the current and undeformed configurations, respectively. Here, the term undeformed refers to the configuration in which appreciable stresses first appear following compaction of a loose packing [1].

We simulate an experiment in which the strains are enforced, and the corresponding stress is computed. To simulate isotropic or polyaxial loading, identical or different normal strains are enforced in three perpendicular directions. A triaxial test is modeled by applying two identical strains in two perpendicular ("lateral") directions. Simulation of uniaxial strain (denoted hereafter as "uniaxial test") mimics loading of a laterally confined pack. We use the convention of positive stress and strain in compression.

2.4.2. Stress. The normal stresses are estimated from the sum of the normal contact forces acting on each boundary, divided by its area [1]. This method is simpler than integrating the product of the force and branch vectors over the sample's volume [35], yet produces very similar results.

The presence of frictional loads implies that the resultant load on the boundaries may include shear and torsion, corresponding to shear stresses. However, in isotropic materials, application of normal principal strains yields normal principal stresses. Thus, if the simulated pack behaves isotropically, the resultant shear and torque must vanish. To verify that no appreciable shear stresses develop, we compute the total moment applied by the normal and tangential contact forces on the boundaries with respect to the pack's center. The averaged shear stress is evaluated from an equivalent force couple. Since this force is at least 2 orders of magnitude smaller than the resultant normal forces, we neglect the shear stresses.

The smallness of the contact area [14] makes the arm of an intergranular torsional couple much smaller than the size of a single grain. Unless torsion acts very close to the pack's center, the torque it applies is negligible relative to that of a contact force. Thus, the contribution of intergranular torsion to the averaged stress is negligible; see also [25].

2.4.3. Elastic moduli. To describe the evolution of the mechanical properties of the pack with loading, we evaluate effective elastic moduli for each load interval. The moduli are found by fitting each stress-strain interval with Hooke's law for a homogeneous, isotropic material [1]. 


\section{SIMULATIONS OF A DEFORMING GRANULAR SAMPLE}

\subsection{Material properties of the grains}

The grain properties for the numerical simulations discussed below are taken from [36, 37]. To model heterogeneous materials such as clastic sediments, we assign the grains radii from a uniform distribution of $0.07-0.13 \mathrm{~mm}$, and elastic moduli from a normal distribution with a standard deviation of $10 \%$ of the mean. A heterogeneous pack of 2740 glass beads (see Figure 3), denoted by G2740, is modeled by assigning the grain moduli with mean values of $\bar{E}=70 \mathrm{GPa}$ and $\bar{\nu}=0.2$, corresponding to $\bar{K}=38.9$ and $\bar{G}=29.2 \mathrm{GPa}$. Here, the overbar denotes the arithmetic mean and $K$ is the bulk modulus. The grain density is set equal to $2.42 \mathrm{~g} / \mathrm{cm}^{3}$. To minimize the deflections of the boundary walls, they are assigned stiffer moduli: $E_{w}=100 \bar{E}$ and $\nu_{w}=0.495$.

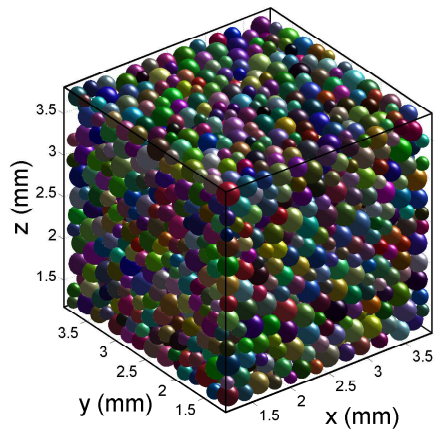

Figure 3. A typical pack used in the simulations with 2740 glass beads, denoted by G2740.

While the grain sizes, densities, and, to some extent, their elastic moduli, can be readily evaluated from experiments, the intergranular friction coefficient, $\mu_{i j}$, is not a well-defined parameter $[38,39]$. For a small contact area, e.g., between slightly deformed spherical grains, the applicability of Coulomb's law of friction is questionable $[38,40]$. The difficulty in estimating the value of $\mu_{i j}$ stems from the fact that intergranular frictional resistance depends on several parameters, such as grain surface roughness, contact area, and velocity, as well as on molecular interactions [40]. As a result, the friction coefficient may vary with the deformation, and with the type of experiment from which it is obtained [39].

Because of the uncertainties associated with the friction coefficient, we run a series of simulations for a range of admissible values, and perform a sensitivity analysis in Section 3.6. For glass beads, values of $0.1-0.3$ were measured [40,41], and used in simulations [6, 10, 40, 42]. In most simulations, we use $\mu=0.3$ for both grain-grain and grain-boundary contacts, where $\mu$ denotes a uniform coefficient for all contacts. Additional simulations using $\mu=0.5$, as well as a pack of 5036 ideally-smooth $(\mu=0)$ glass beads (G5036), are presented.

\subsection{Initial packing}

To make the simulations sensible, the initial grain pack must be sufficiently dense [1]. Such packing can be acquired by tomography imaging of a physical sample, or numerically [1,2]. Here, the numerical procedure to generate such a pack includes two phases: (a) creating a 
dense irregular arrangement, accounting for normal contact forces only [1]; and (b) transition to a frictional model, introducing frictional loads.

The generation procedure starts with a loose irregular arrangement. Here, a loose arrangement is obtained from DEM simulations [43]. In phase (a), the packing density is increased by isotropic compaction of the pack, and expansion of grains with coordination number of $N^{i}<4$, until $N^{i} \geq 4$ for all grains. Note that the grain expansion slightly modifies the originally uniform grain size distribution. Finally, an equilibrium configuration is computed using the frictionless model in [1]. The stress at the end of phase (a) is denoted by $\sigma_{c}^{(\mu=0)}$.

In phase (b), a uniaxial compression of the sample is simulated while accounting for friction. Uniaxial compression causes relative lateral and angular grain displacements, introducing intergranular shear and torsional loads. Simulations of isotropic compression results in smaller grain rearrangements, and thus smaller frictional loads. This result can be explained by the following analogy: in a lattice-type pack, isotropic compression creates self-similar deformation, with only normal compression between the grains. Similar correlation between the degree of loading anisotropy and grain rearrangements is expected in an irregular pack.

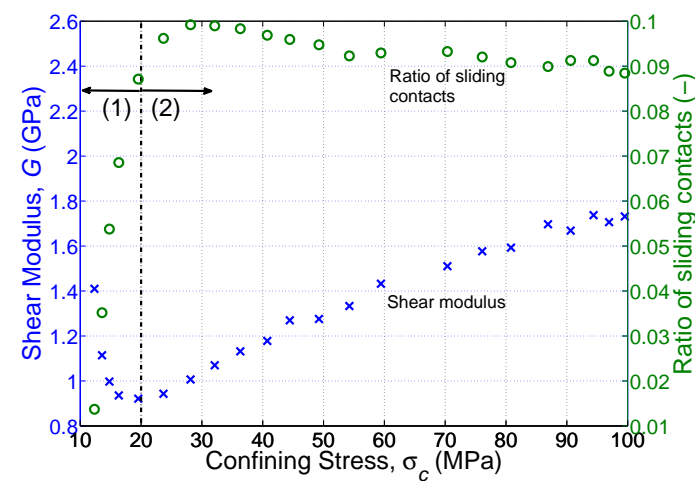

Figure 4. The effective shear modulus, $G$, and ratio of sliding contacts $v s$. confining stress, $\sigma_{c}$, evaluated from sample G2740 with intergranular friction of $\mu=0.3$, during: (1) sample generation procedure, phase (b); and (2) uniaxial test simulation. The two stages are distinct by a dash-dot line.

At the beginning of phase (b), frictional loads rapidly build up. In Figure 4 we plot the computed shear modulus, $G$, vs. the confining stress, $\sigma_{c}=\operatorname{tr}(\boldsymbol{\sigma}) / 3$, where $\operatorname{tr}$ denotes the trace of a tensor. The computed response is stiffer than the one observed experimentally. As phase (b) continues, the initially high $G$ values decrease with $\sigma_{c}$. The sample generation procedure is completed when this abnormal behavior stops: once frictional loads are "well-developed", the moduli increase with $\sigma_{c}$.

The progress of phase (b) is monitored through the relative number of contacts which slide. The ratio of sliding contacts to total number of contacts is plotted in Figure 4. A contact $i j$ is considered sliding if $\left\|\boldsymbol{Q}_{i j}\right\|=Q_{i j}^{\max }$. At the beginning of phase (b), the tangential forces are zero, with no sliding. As the generation procedure continues, the tangential forces increase, initially faster than the normal forces, leading to a rapid increase in the sliding ratio. The shear modulus decreases with the increasing sliding ratio. When the transition to the frictional model is complete, this artificial growth of the tangential forces stops and the sliding ratio becomes relatively constant, $\sim 0.09-0.11$. This "saturation" of the sliding ratio, also observed in the 
simulations reported in $[6,10]$, is the ending criterion for the generation procedure.

\subsection{Verification against experiments}

We test the physical soundness of our model by comparison with published data from acoustic experiments on glass beads ${ }^{\dagger}[36,37,44]$. We note that analytical solutions for the macroscopic parameters are only known for the special case of a lattice-type packing, under the assumption of infinitely-small perturbation [45] or self-similar deformation [2].

Two uniaxial strain tests are simulated using initial packs generated by: (a) isotropic compression of sample 62740 to $\sigma_{c}^{(\mu=0)}=4$ and $\sigma_{c}^{(\mu=0)}=10 \mathrm{MPa}$, followed by (b) uniaxial compression with a friction coefficient of $\mu=0.3$ to $\sigma_{c}=9$ and $\sigma_{c}=19 \mathrm{MPa}$, respectively. Two additional simulations are presented: (1) reproduction of the latter simulation $\left(\sigma_{c}^{(\mu=0)}=10 \mathrm{MPa}\right)$ with $\mu=0.5$; and (2) uniaxial strain applied to sample 65036 with $\mu=0$ [1]. Figure 5 shows the stress-strain curves from which we computed the effective moduli. These curves resemble the experimental and numerical curves presented in [46]. The moduli from our simulations and from the published experiments are plotted in Figure $6 \mathrm{vs}$. confining stress, $\sigma_{c}$. Also plotted are the results of DEM simulations and effective medium theory (EMT) reported in [37] using a contact model that assumes $\mu \rightarrow \infty$.

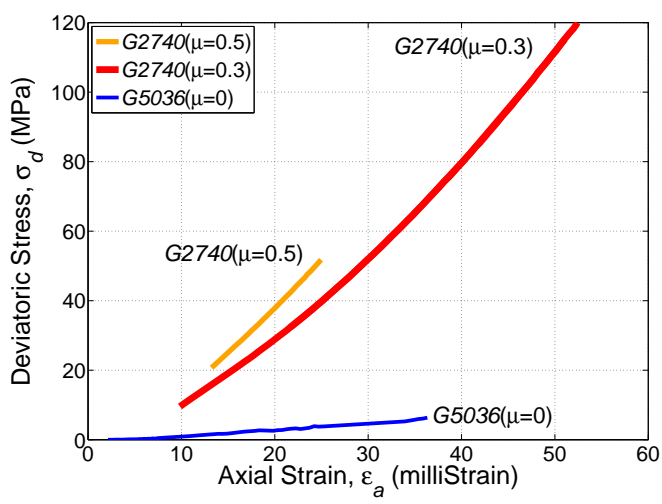

Figure 5. The deviatoric stress, $\sigma_{d}$, computed as the difference between axial and lateral stresses, vs. axial strain, $\epsilon_{a}$, from the simulations on sample G2740 with the intergranular friction coefficient of $\mu=0.3$ and $\mu=0.5$, and on sample $G 5036$ with $\mu=0$ [1]. The stress-strain data from these simulations are used in evaluating the effective moduli presented in Figure 6.

Our estimates and the measured moduli are in good agreement, which has been achieved with no adjustment of material parameters. We stress that the effective moduli are not uniquely defined by the grain properties alone; they also depend on the spatial distribution of these properties and, possibly, on the loading history. Predicting the effective properties is difficult because of the sensitivity of the bulk response to small perturbations, often referred to as "emergent behavior": small variations in grain configurations can result in

$\dagger$ The numerical values of the data from [37,44] have been obtained by digitizing Figure 1 in [37]. The data from [36] are computed from the velocities reported by the author, see [2]. 

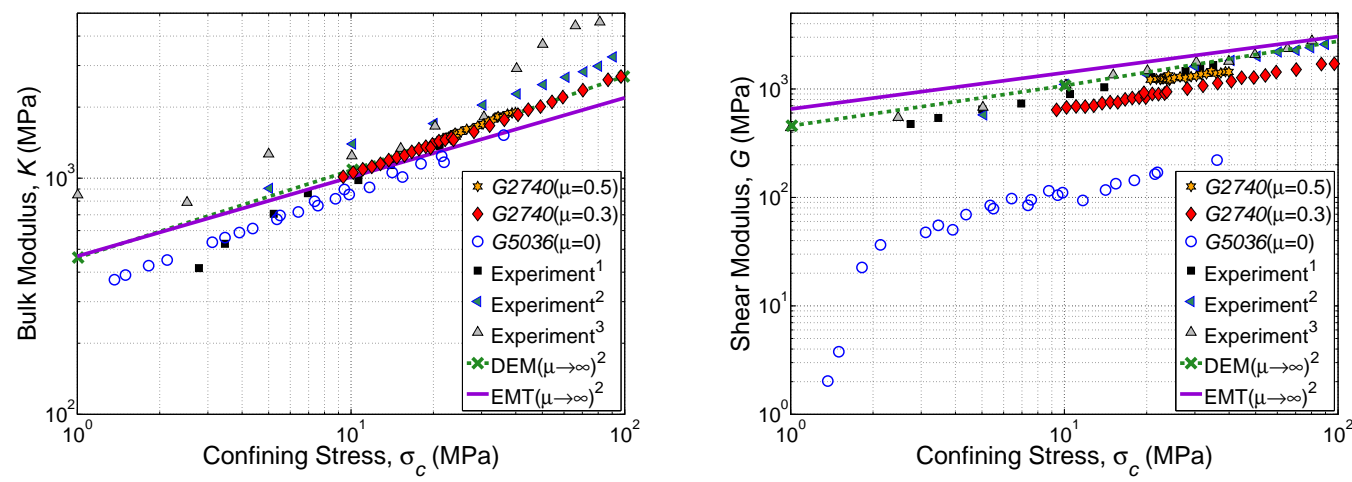

Figure 6. The effective bulk modulus $\left(K\right.$, left) and shear modulus ( $G$, right) vs. confining stress, $\sigma_{c}$. The moduli evaluated from the simulations on sample G2740 with intergranular friction coefficient of $\mu=0.3$ and $\mu=0.5$, and on sample G5036 using a frictionless model $(\mu=0)$ [1], are compared with the results of acoustic experiments in glass beads, as well as DEM and EMT predictions using $\mu \rightarrow \infty$. Superscripts 1-3 refer to the data published in [36], [37], and [44], respectively. Good agreement between our estimates and the experimental data, achieved with no adjustment of material parameters, demonstrates the physical soundness of our model.

significantly different properties ([1,2,38] and the references therein). Inevitable uncertainty in characterizing the grain configurations and the loading history of each contact results in large scatter in experimental data, e.g., see Figure 6, making exact reproduction of an experiment by numerical simulations impossible.

Our simulations show a gradual decrease in the rate of moduli growth with confining stress, from a power law of $\sigma_{c}^{0.45}$ to $\sigma_{c}^{0.33}$. This transition, while observed in experiments and justified by theory, is not predicted by the classical EMT [47]. Our model provides additional theoretical interpretation for this transition. Increasing the confining stress increases both the packing density and stiffness, while decreasing their growth rates. As the pack gets denser, further compaction becomes more difficult. The relation between the contact stiffness and $\sigma_{c}$ can be predicted from the Hertzian contact law, $c f$. Eq. (2): the normal contact stiffness is the derivative of the force magnitude, $\left\|\boldsymbol{P}_{i j}\right\|$, with respect to the deformation, $h_{i j}$, where the latter is expected to increase with the confining stress. Therefore, the growth rate of the normal stiffness is proportional to the second derivative of $\left\|\boldsymbol{P}_{i j}\right\|$ with a power law of $\left(h_{i j}\right)^{-1 / 2}$, which indicates an inverse relation between the stiffness and $\sigma_{c}$. Since the moduli increase with both the packing density and the contact stiffness, the transition in power law is expected.

\subsection{Sensitivity of the effective moduli to the initial configuration}

The sample generation procedure in Section 3.2 involves "activation" of frictional resistance through uniaxial loading, which can affect the sample properties. In this section, we examine the effect of the initial packing on the effective moduli. By the term "initial packing" we refer to the configuration of the grains, the intergranular loads, and the sample generation procedure.

To generate a number of different initial packs, sample G2740 has been compacted isotropically up to stresses of $\sigma_{c}^{(\mu=0)}=4,10,20,41,54$ and $74 \mathrm{MPa}$ in phase (a), followed by application of uniaxial strain with a friction coefficient of $\mu=0.3$ in phase (b). The resulting 
initial packs are denoted by G2740(4), G2740(10), G2740(20), G2740(41), G2740(54), and G2740(74), respectively. Although the packs are composed of assemblies of identical grains, the microstructure of the samples, and consequently their mechanical properties, may differ due to different loading paths [48].
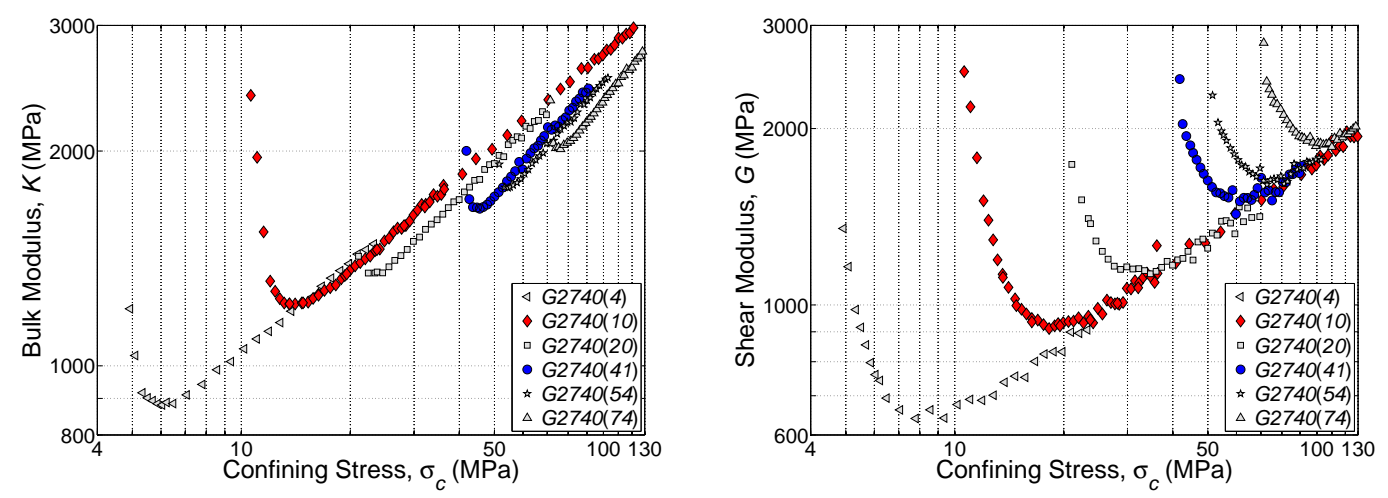

Figure 7 . The effective bulk modulus $\left(K\right.$, left) and shear modulus ( $G$, right) vs. confining stress, $\sigma_{c}$, evaluated during: (1) sample generation procedure, phase (b); and (2) uniaxial compression test simulations using sample G2740 with a friction coefficient of $\mu=0.3$. Six simulations were conducted on initial packs generated with different stresses during phase $(\mathrm{a}), \sigma_{c}^{(\mu=0)}$. At a given $\sigma_{c}, K$ is lower for samples with higher $\sigma_{c}^{(\mu=0)}$ values, e.g. it is lower for G2740(41) than for $62740(10)$, whereas $G$ shows less sensitivity to $\sigma_{c}^{(\mu=0)}$.

Figure 7 shows the effective moduli of these packs plotted vs. confining stress, $\sigma_{c}$, evaluated during: (1) phase (b) of the generation procedure; and (2) uniaxial test simulation. Comparing the moduli evaluated at similar confining stresses, we observe that the bulk modulus is lower for samples with higher $\sigma_{c}^{(\mu=0)}$ values. While the difference between the bulk modulus evaluated for G2740(4) and G2740(10) is only 1\%, the difference with other samples, e.g. G2740(41), increases to $\sim 10 \%$. The shear modulus estimates are less sensitive to the initial pack, with relatively small variations among the different simulations.

To interpret these seemingly counterintuitive observations, we calculate the mean normal contact force within the pack, $\bar{P}$. At a given confining stress, $\bar{P}$ is found to be higher in packs with lower $\sigma_{c}^{(\mu=0)}$. This difference reflects the different load paths in the generation procedure: for a sample with lower $\sigma_{c}^{(\mu=0)}$, uniaxial strain loading starts at a lower stress, indicating larger axial stress and lower lateral stress. As discussed in Section 3.2, stronger loading anisotropy results in more grain rearrangements. Rearrangements increase the packing density, and consequently the growth rate of the normal contact forces. Since the bulk modulus is mainly affected by the normal contact forces [1], it increases with $\bar{P}$, while the shear modulus, which strongly depends on the intergranular friction, shows smaller sensitivity.

\subsection{Sensitivity to the size of the load increments}

The tradeoff between the size of the load increments and the total number of increments imposes constraints on the increment size. To examine sensitivity of the model to the increment size, we compare the simulations on sample G2740, applying different strain 
increments, $\Delta \epsilon_{a}$. In Figure 8, the moduli evaluated during: (1) phase (b) of the generation procedure, and (2) uniaxial test simulation, are plotted $v s$. confining stress. We present results from two simulations using $\Delta \epsilon_{a}=5 \cdot 10^{-5}$ and $\Delta \epsilon_{a}=5 \cdot 10^{-4}$, in addition to a simulation in which the strain increments were reduced from $\Delta \epsilon_{a}=5 \cdot 10^{-4}$ during the generation procedure to $\Delta \epsilon_{a}=5 \cdot 10^{-5}$ during the uniaxial test. The similarity of the curves implies that the results are robust with respect to $\Delta \epsilon_{a}$. In the simulations presented in the previous sections, increments of $\Delta \epsilon_{a}=10^{-4}$ or smaller were used.
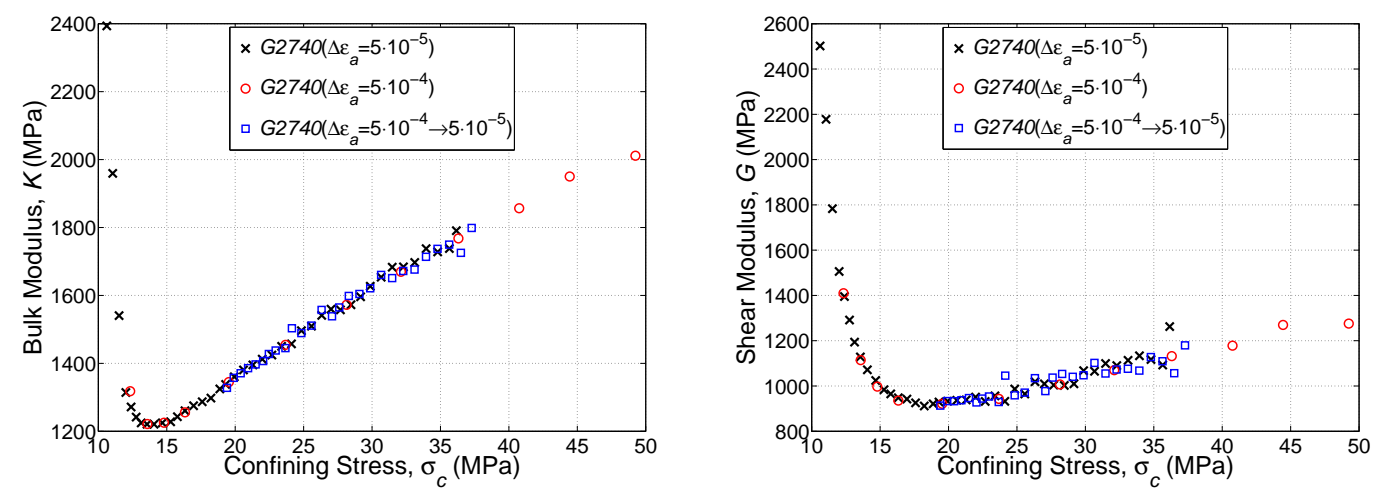

Figure 8. The effective bulk modulus ( $K$, left) and shear modulus ( $G$, right) vs. confining stress, $\sigma_{c}$, evaluated during: (1) sample generation procedure, phase (b); and (2) uniaxial compression test simulations using sample 62740 with a friction coefficient of $\mu=0.3$. We compare three simulations, enforcing strain increments of $\Delta \epsilon_{a}=5 \cdot 10^{-4}, \Delta \epsilon_{a}=5 \cdot 10^{-5}$, and their combination. The small differences demonstrate the robustness of the results with respect to the size of the load increments.

\subsection{Effect of the intergranular friction coefficient}

The effects of the intergranular friction on the bulk response, the microstructure, and the spatial distribution of the contact forces have been studied in $[6,10,38,39,42,49,50]$. For example, experiments and simulations show that the shearing resistance correlates with the intergranular friction coefficient $[10,39,49,50]$. Note that intergranular frictional resistance is not uniquely determined by the friction coefficient. In our model, this resistance is associated with: (1) the friction coefficient, $\mu$, and the magnitude of the normal force, $\left\|\boldsymbol{P}_{i j}\right\|$, if sliding occurs; and (2) the tangential and torsional stiffnesses, $k_{i j(s)}$ and $k_{i j(t)}$, if the contact does not slide. The sliding threshold, $Q_{i j}^{\max }$, and, on the verge of sliding, the reduced stiffness values, $k_{i j(s)}^{\prime}$ and $k_{i j(t)}^{\prime}$, depend on $\mu$ and $\left\|\boldsymbol{P}_{i j}\right\|$.

The sensitivity of the effective moduli to the friction coefficient is investigated by comparing the moduli predicted from two uniaxial test simulations $\left(\sigma_{c}^{(\mu=0)}=10 \mathrm{MPa}\right)$, using $\mu=0.3$ and $\mu=0.5$, see Figure 9. As expected, the moduli, in particular the shear modulus, increase with the friction coefficient. The bulk modulus is less sensitive to the intergranular friction.

To complete this analysis, we study the effect of extreme $\mu$ values. A frictionless model $(\mu=0)$ underestimates the stiffness and strength of the pack, in particular under shear; for example, it predicts loss of shear rigidity, $G \rightarrow 0$, at relatively high packing densities $[1,37] ; c f$. Figure 6 . Conversely, a model which prohibits sliding by assuming $\mu \rightarrow \infty$ overestimates $G$ [37]. 

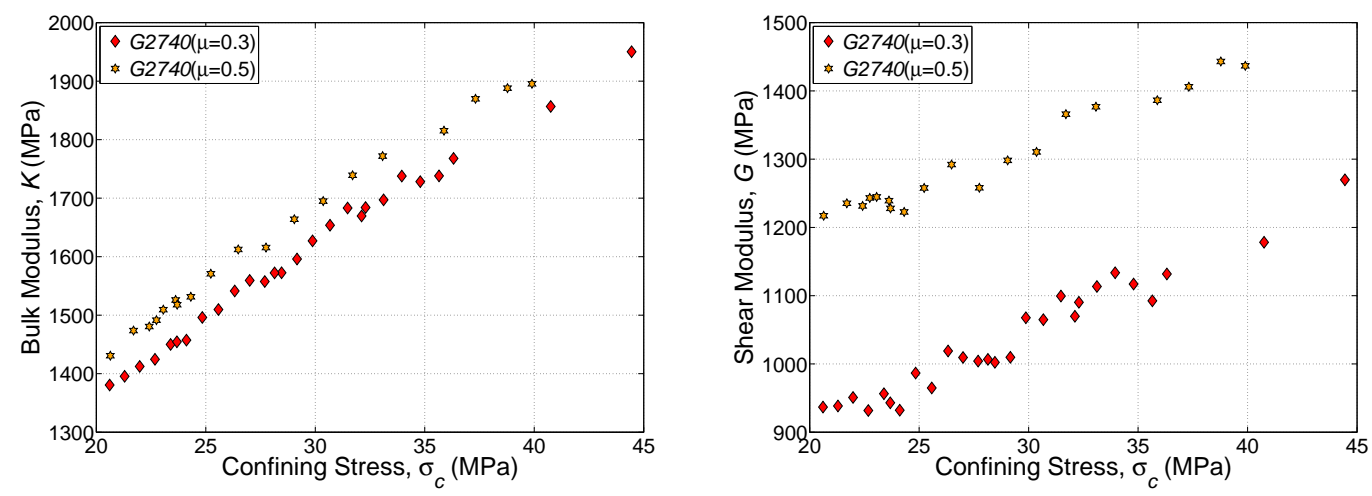

Figure 9. The effective bulk modulus ( $K$, left) and shear modulus ( $G$, right) vs. confining stress, $\sigma_{c}$, evaluated from two simulations on sample G2740, using different intergranular friction coefficients, $\mu=0.3$ and $\mu=0.5$. The effective moduli, in particular $G$, increase with the friction coefficient.

Note that macroscopic stiffness and strength do not increase significantly when the friction coefficient increases above a certain value (e.g., $\mu=0.6$ in [42]). Similar saturation of the contact force distribution and the mean coordination number with respect to $\mu$ has been observed in simulations [6] and experiments [51].

\section{SUMMARY AND CONCLUDING REMARKS}

A micromechanical model of a cohesionless particulate material with intergranular friction has been presented. We simulate the deformation and evaluate the macroscopic elastic properties of a granular pack using a computational technique denoted by QuSGM. In QuSGM, a threedimensional, irregular pack of spherical grains is loaded by incremental displacement of its boundaries. Deformation is described by a sequence of static equilibrium configurations. The intergranular interactions, including friction and sliding, are modeled by a set of constitutive relations based on the contact theories of Hertz, Mindlin, and Deresiewicz. The macroscopic stress, strain and elastic moduli are evaluated from the intergranular forces and the deformation of the pack. Based on the principle of least work, a variational approach is employed to find the equilibrium configurations by minimizing the work against the loads acting on the grains. This minimum is computed using a modified conjugate gradient algorithm.

Our model has been verified by assigning experimentally measured grain properties, and comparing the calculated effective moduli with published experiments. Good agreement between predicted and measured moduli, achieved with no adjustment of material parameters, establishes the physical soundness of the quasi-static approach. In addition, the predicted moduli are shown to be robust with respect to the size of the load increment. Our approach does not involve numerical damping, which is often used in dynamic models such as the discrete element method.

QuSGM is used to generate a sufficiently dense, irregular packing. Simulations show that the bulk modulus estimates are more sensitive to the initial pack formation procedure than the shear modulus. Thus, the effective elastic moduli are not uniquely defined by the grain 
properties alone; they also depend on the spatial distribution of these properties and on the loading history. This indeterminacy is enhanced by the sensitivity of the bulk response to small variations in grain configurations. Uncertainty in experimental characterization of the grain configurations and loading history prevents an exact numerical reproduction of an experiment, and is associated with the scatter in experimental data.

Sensitivity analysis confirms that the stiffness and strength of a granular pack increase with the intergranular friction coefficient. The shear modulus is more sensitive to the intergranular friction than the bulk modulus, which mainly depends on the normal contact forces. We conclude that proper account of intergranular frictional resistance is required to predict the macroscopic response, in particular under shear.

In summary, QuSGM is a useful tool to advance the understanding of granular mechanics. Our simulations complement physical testing of granular materials, and develop insights into the design of new experiments. Elsewhere [1,2], we have applied our approach to model cohesionless and weakly-cemented sediments, accounting for hydrate dissociation and cementation of grain contacts. The current model can be extended to account for inelastic grain deformations (e.g., [52]), as well as for irregular grains that can fracture or break. The irregular grains can be modeled as clusters of spherical grains bonded at their contacts [53]. Such extensions will allow QuSGM to elucidate the micromechanics of a wide range of complex geological systems.

\section{APPENDIX A: NUMERICAL ALGORITHM}

This appendix outlines the numerical algorithm used in the simulations. Following each load increment, simulated through displacements of the pack boundaries, a modified conjugate gradient (CG) algorithm [54] is employed to find a new (current) equilibrium configuration of the grains. The algorithm searches for a local minimum of an energy functional, П. According to the principle of least work [13], $\Pi$ is computed from the work against the intergranular loads, cf. Eqs. (14)-(17). The force and moment balance equations, Eqs. (13), are nonlinear, and $\Pi$ is not quadratic, for the following reasons: (a) nonlinearity of the intergranular constitutive relations, and (b) variations in the contact network. The modifications of the classical CG algorithm to accommodate the nonlinearity of the balance equations are summarized below. For further discussion on implementation of CG in granular mechanics, see [55].

\section{A.1. Initial Guess}

The iterative minimization procedure begins with an "initial guess": here, $\boldsymbol{\theta}^{k=0}=\mathbf{0}$ is chosen, where superscript $k$ denotes the iteration. We use a fixed Cartesian coordinate system aligned with the pack's boundaries, with an origin at the pack's geometric center, $\boldsymbol{x}_{C M}$. Application of each load increment is modeled by symmetric displacements of each pair of parallel boundaries, $\boldsymbol{u}_{w=l}=-\boldsymbol{u}_{w=l+3}$, where $l=1,2,3$ denotes the coordinate directions. These displacements are applied in several sub-increments, every $k_{i n c}$ iterations, see Figure A1. After each incremental boundary displacement, we select a new initial guess for the grain displacements and positions: for each direction, $l$, the update for grain $i$ is computed from the displacement of the boundaries 
scaled by the ratio between the distance of the grain to the pack's center and the side length,

$$
\Delta \boldsymbol{u}_{i(l)}^{k}=\frac{\left|\left(\boldsymbol{x}_{C M}-\boldsymbol{r}_{i}^{k}\right) \cdot \hat{\boldsymbol{e}}_{l}\right|}{2 L_{l}^{k}} \Delta \boldsymbol{u}_{w=l}^{k}
$$

where $L_{l}, \Delta \boldsymbol{u}_{w=l}^{k}$, and $\hat{\boldsymbol{e}}_{l}$ are the pack's length, the incremental displacement, and a unit vector associated with the nearest boundary in the $l$ direction, respectively. The incremental boundary displacements, $\Delta \boldsymbol{u}_{l}^{k}$, are applied until the total desired boundary displacement for that load increment, $\boldsymbol{u}_{w=l}$, has been achieved.

1. Initial guess $(k=0): \boldsymbol{\theta}^{k=0}=\mathbf{0}$. Set $\boldsymbol{p}^{k=0}=\left.\nabla_{\boldsymbol{\theta}} \Pi\right|_{\boldsymbol{\theta}^{k=0}}$.

2. IF $k$ divisible by $k_{i n c}$ AND $\boldsymbol{u}_{w}^{k}<\boldsymbol{u}_{w}$, apply sub-incremental perturbation:

(a) Update the boundary positions: $\boldsymbol{x}_{w}^{k} \leftarrow \boldsymbol{x}_{w}^{k}+\Delta \boldsymbol{u}_{w}^{k}$ and $\boldsymbol{u}_{w}^{k} \leftarrow \boldsymbol{u}_{w}^{k}+\Delta \boldsymbol{u}_{w}^{k}$

(b) Update the grain displacements ("initial guess"), $\boldsymbol{u}_{i}^{k} \leftarrow \boldsymbol{u}_{i}^{k}+\Delta \boldsymbol{u}_{i}^{k}$ and positions, $\boldsymbol{r}_{i}^{k} \leftarrow \boldsymbol{r}_{i}^{k}+\Delta \boldsymbol{u}_{i}^{k}, c f$. Section A.1.

3. Evaluate $\left.\nabla_{\boldsymbol{\theta}} \Pi\right|_{\boldsymbol{\theta}^{k}}, c f$. Eq. (18).

4. IF $k=1$ OR $k$ divisible by $k_{S D}$, refresh with a steepest descent step: set $\beta^{k}=0$. $\operatorname{ELSE} \beta^{k}=-\left(\frac{\left\|\left.\nabla_{\boldsymbol{\theta}} \Pi\right|_{\boldsymbol{\theta}^{k}}\right\|}{\left\|\left.\nabla_{\boldsymbol{\theta}} \Pi\right|_{\boldsymbol{\theta}^{k-1}}\right\|}\right)^{2}$.

5. Compute the "search direction" vector, $\boldsymbol{p}^{k}=\left.\nabla_{\boldsymbol{\theta}} \Pi\right|_{\boldsymbol{\theta}^{k}}-\beta^{k} \boldsymbol{p}^{k-1}$.

6. Given $\boldsymbol{\theta}^{k}$ and $\boldsymbol{p}^{k}$, find $\alpha^{k}$ that minimizes $\Pi\left(\boldsymbol{\theta}^{k}-\alpha^{k} \boldsymbol{p}^{k}\right)$, cf. Section A.2.

7. Update the generalized coordinates, $\boldsymbol{\theta}^{k+1}=\boldsymbol{\theta}^{k}-\alpha^{k} \boldsymbol{p}^{k}$, and the grain positions, $\boldsymbol{r}_{i}^{k+1}=\boldsymbol{r}_{i}^{k}+\boldsymbol{u}_{i}^{k}$.

8. Check convergence:

IF $\Pi\left(\boldsymbol{x}^{k-1}\right)-\Pi\left(\boldsymbol{x}^{k}\right)<\tau_{1}\left|\Pi\left(\boldsymbol{x}^{k-1}\right)\right|$ OR $\left\|\left.\nabla_{\boldsymbol{\theta}} \Pi\right|_{\boldsymbol{\theta}^{k}}\right\|^{2}<\tau_{2} N\left(\bar{E} \bar{R}^{2}\right)^{2}$ OR $k>k_{\max }$ STOP.

ELSE, set $k \leftarrow k+1$ and GOTO Step 2 .

Figure A1. A modified conjugate gradient algorithm used to simulate application of a load increment, and to obtain a subsequent equilibrium configuration.

\section{A.2. Iterative Update}

Since the criterion $\Pi$ is not quadratic, the update coefficient $\alpha^{k}$ is found numerically by minimizing $\Pi\left(\boldsymbol{\theta}^{k}-\alpha^{k} \boldsymbol{p}^{k}\right)$ with respect to $\alpha^{k}$. We perform this minimization using the MATLAB function fminbnd [56], which utilizes the golden section search and parabolic interpolation methods [54]. To avoid numerical artifacts in the form of grain permutations, which correspond to a non-local minimum of $\Pi$, we restrict the update of the grain displacements within each iteration. This restriction is enforced by limiting the value of $\alpha$ so that the displacement of any grain does not exceed $10^{-3}-10^{-1}$ of its radius. The optimal constraint value is found by trial and error. 


\section{A.3. Convergence Criterion}

Theoretically, the CG method yields an exact solution to a system of linear equations with a symmetric, positive-definite matrix within a number of iterations that does not exceed the dimensions of the system, $N$ [54]. Here, due to the nonlinearity of the balance equations, the algorithm may not work in the same manner as it does for a linear system of equations [55]. To avoid loss of conjugacy, the iterative procedure is periodically refreshed every $k_{S D}$ iterations by setting $\beta^{k}=0$, equivalent to performing a steepest descent (SD) step, see Figure A1. The frequency of this operation is determined by trial and error.

The iterations stop if convergence has been achieved within a given tolerance, specified by

$$
\Pi^{k-1}-\Pi^{k}<\tau_{1}\left|\Pi\left(\boldsymbol{x}^{k-1}\right)\right| \quad \text { or } \quad\left\|\left.\nabla_{\boldsymbol{\theta}} \Pi\right|_{\boldsymbol{\theta}^{k}}\right\|^{2}<\tau_{2} N\left(\bar{E} \bar{R}^{2}\right)^{2}
$$

where $\bar{R}$ and $\bar{E}$ are the mean grain radius and Young's modulus. For example, for sample G2740 with $\bar{E}=7 \cdot 10^{10} \mathrm{~Pa}$ and $\bar{R}=10^{-4} \mathrm{~m}$, the tolerance parameters are $\tau_{1}=10^{-11}$ and $\tau_{2}=10^{-13}$. These values provide for each grain a residual force, $\boldsymbol{F}_{i}$, and moment, $\boldsymbol{M}_{i}$, which are smaller than the maximum contact force and moment on that grain by at least 4 orders of magnitude. Formally, to avoid infinite cycling, the number of iterations is constrained by $k_{\max }$. Applying a strain increment of $\Delta \epsilon_{a}=10^{-4}$ with $k_{i n c}=10$, the number of iterations required to find an equilibrium configuration is $100-130$.

The algorithm in Figure A1 was coded in MATLAB [56]. The typical running time to simulate the application of $\Delta \epsilon_{a}=10^{-4}$ on sample G2740 is $\sim 1$ hour, using a standard desktop with Intel Pentium D 2.80 GHz processor and 2.00GB of RAM. In [2], the performance of the code has been evaluated by comparing the numerical prediction with an analytical solution for the special case of a small, structured packing undergoing self-similar deformations. The relative error, measured as the deviation from a self-similar deformation, is $\sim 10^{-11}-10^{-9}$.

\section{ACKNOWLEDGEMENT}

This work was partially supported by the Assistant Secretary for Fossil Energy, Office of Natural Gas and Petroleum Technology, through the National Energy Technology Laboratory, under the U.S. Department of Energy, Contract \#DE-FC26-05NT42664. Additional support through U.C. Oil Consortium is greatly appreciated. We thank the editor and the reviewers for their critical comments.

\section{REFERENCES}

1. Holtzman R, Silin DB, Patzek TW. Mechanical properties of granular materials: a variational approach to grain-scale simulations. International Journal for Numerical and Analytical Methods in Geomechanics 2009; 33(3):391-404, DOI: 10.1002/nag.725.

2. Holtzman R. Micromechanics of sediments: a variational approach to grain-scale simulations. PhD Thesis, University of California, Berkeley, CA 2008.

3. Cundall PA, Strack ODL. A discrete numerical model for granular assemblies. Géotechnique 1979; 29:4765.

4. O'Sullivan C, Bray JD. Selecting a suitable time-step for discrete element simulations that use the central difference time integration approach. Engineering Computations 2004; 21(2-4):278-303, DOI: 10.1108/02644400410519794.

5. Tavarez FA, Plesha ME. Discrete element method for modelling solid and particulate materials. International Journal for Numerical Methods in Engineering 2007; 70:379-404, DOI: 10.1002/nme.1881. 
6. Thornton C. Numerical simulations of deviatoric shear deformation of granular media. Géotechnique 2000; 50(1):43-53

7. O'Sullivan C, Bray JD, Riemer M. Examination of the response of regularly packed specimens of spherical particles using physical tests and discrete element simulations. Journal of Engineering Mechanics 2004; 130(10):1140-1150, DOI: 10.1061/(ASCE)0733-9399(2004)130:10(1140).

8. Potyondy DO, Cundall PA. A bonded-particle model for rock. International Journal of Rock Mechanics and Mining Sciences 2004; 41(8):1329-1364, DOI: 10.1016/j.ijrmms.2004.09.011.

9. Tavarez FA. Discrete element method for modelling solid and particulate materials. PhD Thesis, University of Wisconsin, Madison, WI 2005.

10. Peña AA, Lizcano A, Alonso-Marroquin F, Herrmann HJ. Biaxial test simulations using a packing of polygonal particles. International Journal for Numerical and Analytical Methods in Geomechanics 2008; 32(2):143-160, DOI: $10.1002 /$ nag.618.

11. Thomas PA, Bray JD. Capturing nonspherical shape of granular media with disk clusters. Journal of Geotechnical and Geoenvironmental Engineering 1999; 125(3):169-178, DOI: 10.1061/(ASCE)10900241(1999)125:3(169).

12. Shi GH. Discontinuous deformation analysis: A new numerical model for the statics and dynamics of block systems. PhD Thesis, University of California, Berkeley, CA 1988.

13. Timoshenko SP, Goodier JN. Theory of Elasticity. Engineering societies monographs, McGraw-Hill: New York, 1970.

14. Johnson KL. Contact Mechanics. Cambridge University Press: Cambridge, MA, 1987.

15. Dintwa E, Tijskens E, Ramon H. On the accuracy of the Hertz model to describe the normal contact of soft elastic spheres. Granular Matter 2008; 10(3):209-221, DOI: 10.1007/s10035-007-0078-7.

16. Kruyt NP, Antony SJ. Force, relative-displacement, and work networks in granular materials subjected to quasistatic deformation. Physical Review E 2007; 75(5):051308, DOI: 10.1103/PhysRevE.75.051308.

17. Goldenhirsch I, Goldenberg C. Stress in dense granular materials. The Physics of Granular Media, Hinrichsen H, Wolf DE (eds.). Wiley-VCH, 2005.

18. Mindlin RD, Deresiewicz H. Elastic spheres in contact under varying oblique forces. Journal of Applied Mechanics 1953; 20:327-344.

19. Deresiewicz H. Contact of elastic spheres under an oscillating torsional couple. Journal of Applied Mechanics 1954; 21:52-56.

20. Vu-Quoc L, Zhang X. An accurate and efficient tangential force-displacement model for elastic frictional contact in particle-flow simulations. Mechanics of Materials 1999; 31(4):235-269, DOI: 10.1016/S01676636(98)00064-7.

21. Dobry R, Ng TT, Petrakis E, Seridi A. General model for contact law between two rough spheres. Journal of Engineering Mechanics 1991; 117(6):1365-1381, DOI: 10.1061/(ASCE)0733-9399(1991)117:6(1365).

22. Segalman DJ, Starr MJ, Heinstein MW. New approximations for elastic spheres under an oscillating torsional couple. Journal of Applied Mechanics 2005; 72(5):705-710, DOI: 10.1115/1.1985430.

23. Elata D, Berryman JG. Contact force-displacement laws and the mechanical behavior of random packs of identical spheres. Mechanics of Materials 1996; 24(3):229-240, DOI: 10.1016/S0167-6636(96)00034-8.

24. Walton OR, Braun RL. Viscosity, granular-temperature, and stress calculations for shearing assemblies of inelastic, frictional disks. Journal of Rheology 1986; 30(5):949-980, DOI: 10.1122/1.549893.

25. Goddard JD. Nonlinear elasticity and pressure-dependent wave speeds in granular media. Proceedings of the Royal Society of London, Series A 1990; 430:105-131, DOI: 10.1098/rspa.1990.0083.

26. Deresiewicz H. Mechanics of granular matter. Advances in Applied Mechanics 1958; 5:233-306.

27. Vaisnys JR, Pilbeam CC. Mechanical properties of granular media. Annual Review of Earth and Planetary Sciences 1975; 3(1):343-360, DOI: 10.1146/annurev.ea.03.050175.002015.

28. O'Sullivan C. The application of discrete element modelling to finite deformation problems in geomechanics. PhD Thesis, University of California, Berkeley, CA 2002.

29. Bagi K. From order to chaos: the mechanical behaviour of regular and irregular assemblies. QuaDPM'03 Workshop, 25-28 August 2003, Bagi K (ed.), Budapest, Hungary, 2003; 33-42.

30. Walton OR. Numerical simulation of inclined chute flows of monodisperse, inelastic, frictional spheres. Mechanics of Materials 1993; 16(1-2):239-247.

31. Norris AN, Johnson DL. Nonlinear elasticity of granular media. Journal of Applied Mechanics 1997; 64:39-49, DOI: 10.1115/1.2787292.

32. Lubkin JL. The torsion of elastic spheres in contact. Journal of Applied Mechanics 1951; 18:183-187.

33. Hills DA. The stress field induced by a twisting sphere. Journal of Applied Mechanics 1986; 53:372-378.

34. Landau LD, Lifshitz EM. Theory of Elasticity. Course of Theoretical Physics, Vol 7, Elsevier: Burlington, MA, 1986.

35. Christoffersen J, Mehrabadi MM, Nemat-Nasser S. A micromechanical description of granular material behavior. Journal of Applied Mechanics 1981; 48(2):339-344.

36. Domenico SN. Elastic properties of unconsolidated porous sand reservoirs. Geophysics 1977; 42(7):1339- 
1368, DOI: $10.1190 / 1.1440797$.

37. Makse HA, Gland N, Johnson DL, Schwartz LM. Why effective medium theory fails in granular materials. Physical Review Letters 1999; 83(24):5070-5073, DOI: 10.1103/PhysRevLett.83.5070.

38. Behringer RP, Howell D, Kondic L, Tennakoon S, Veje C. Predictability and granular materials. Physica D 1999; 133(1-4):1-17, DOI: 10.1016/S0167-2789(99)00094-9.

39. Pohlman NA, Severson BL, Ottino JM, Lueptow RM. Surface roughness effects in granular matter: influence on angle of repose and the absence of segregation. Physical Review E 2006; 73(3):031304, DOI: 10.1103/PhysRevE.73.031304.

40. Li Y, Xu Y, Thornton C. A comparison of discrete element simulations and experiments for 'sandpiles' composed of spherical particles. Powder Technology 2005; 160(3):219-228, DOI: 10.1016/j.powtec.2005.09.002.

41. Klaas NV, Marcus K, Kellock C. The tribological behaviour of glass filled polytetrafluoroethylene. Tribology International 2005; 38(9):824-833, DOI: 10.1016/j.triboint.2005.02.010.

42. Muthuswamy M, Tordesillas A. How do interparticle contact friction, packing density and degree of polydispersity affect force propagation in particulate assemblies? Journal of Statistical Mechanics: Theory and Experiment 2006; 2006(09):P09 003, DOI: 10.1088/1742-5468/2006/09/P09003.

43. Jin G. Physics-based modeling of sedimentary rock formation and prediction of transport properties. PhD Thesis, University of California, Berkeley, CA 2006.

44. Yin H. Acoustic velocity and attenuation of rocks: Isotropy, intrinsic anisotropy, and stress-induced anisotropy. PhD Thesis, Stanford University, Stanford, CA 1993.

45. Deresiewicz H. Stress-strain relations for a simple model of a granular medium. Journal of Applied Mechanics 1958; 25:402-406.

46. Holt RM, Kjølaas J, Larsen I, Li L, Gotusso Pillitteri A, Sønstebø EF. Comparison between controlled laboratory experiments and discrete particle simulations of the mechanical behaviour of rock. International Journal of Rock Mechanics and Mining Sciences 2005; 42(7-8):985-995, DOI: 10.1016/j.ijrmms.2005.05.006.

47. Pride SR. Relationships between seismic and hydrological properties. Hydrogeophysics, Rubin Y, Hubbard S (eds.). Springer: Dordrecht, The Netherlands, 2005.

48. Magnanimo V, La Ragione L, Jenkins JT, Wang P, Makse HA. Characterizing the shear and bulk moduli of an idealized granular material. Europhysics Letters 2008; 81(3):34 006, DOI: 10.1209/0295-5075/81/34006.

49. Anthony JL, Marone C. Influence of particle characteristics on granular friction. Journal of Geophysical Research 2005; 110:B08 409, DOI: 10.1029/2004JB003399.

50. Cui L, O'Sullivan C. Exploring the macro- and micro-scale response of an idealised granular material in the direct shear apparatus. Géotechnique 2006; 56(7):455-468, DOI: 10.1680/geot.56.7.455.

51. Blair DL, Mueggenburg NW, Marshall AH, Jaeger HM, Nagel SR. Force distributions in threedimensional granular assemblies: effects of packing order and interparticle friction. Physical Review $E$ 2001; 63(4):041 304, DOI: 10.1103/PhysRevE.63.041304.

52. Zhang X, Vu-Quoc L. An accurate elasto-plastic frictional tangential force-displacement model for granularflow simulations: displacement-driven formulation. Journal of Computational Physics 2007; 225:730-752, DOI: $10.1016 /$ j.jcp.2006.12.028.

53. Vu-Quoc L, Zhang X, Walton OR. A 3-D discrete-element method for dry granular flows of ellipsoidal particles. Computer Methods in Applied Mechanics and Engineering 2000; 187(3-4):483-528, DOI: 10.1016/S0045-7825(99)00337-0.

54. Press WH, Flannery BP, Teukolsky SA, Vetterling WT. Numerical Recipes in Fortran 77. Cambridge University Press: New York, 1986.

55. Renouf M, Alart P. Conjugate gradient type algorithms for frictional multi-contact problems: applications to granular materials. Computer Methods in Applied Mechanics and Engineering 2005; 194:2019-2041, DOI: $10.1016 /$ j.cma.2004.07.009

56. The MathWorks Inc. MATLAB, The Language of Technical Computing, Version 7.6.0.324 (R2008a). 2008. 